\title{
Short communication: Conservation of Streptococcus uberis adhesion molecule and the sua gene in strains of Streptococcus uberis isolated from geographically diverse areas
}

\author{
Ying Yuan, ${ }^{*}$ Oudessa Kerro Dego, $†$ Xueyan Chen, $\dagger$ Eurife Abadin,‡ Shangfeng Chan, ${ }^{*}$ Lauren Jory, $\ddagger$ \\ Steven Kovacevic, $\ddagger$ Raul A. Almeida, ${ }^{1}$ and Stephen P. Oliver \\ *Zoetis, Veterinary Medicine Research and Development (VMRD), Global Therapeutic Research, 333 Portage Street, Kalamazoo, MI 49007 \\ †Department of Animal Science, The University of Tennessee, Knoxville 37996 \\ łZoetis, VMRD, Global Therapeutic Research, 405 Poplar Road, Parkville, Victoria 3052, Australia
}

\begin{abstract}
The objective was to identify and sequence the sua gene (GenBank no. DQ232760; http://www.ncbi.nlm. nih.gov/genbank/) and detect Streptococcus uberis adhesion molecule (SUAM) expression by Western blot using serum from naturally $S$. uberis-infected cows in strains of $S$. uberis isolated in milk from cows with mastitis from geographically diverse areas of the world. All strains evaluated yielded a $4.4-\mathrm{kb}$ sua-containing PCR fragment that was subsequently sequenced. Deduced SUAM AA sequences from those $S$. uberis strains evaluated shared $>97 \%$ identity. The pepSUAM sequence located at the N terminus of SUAM was $>99 \%$ identical among strains of S. uberis. Streptococcus uberis adhesion molecule expression was detected in all strains of S. uberis tested. These results suggest that sua is ubiquitous among strains of $S$. uberis isolated from diverse geographic locations and that SUAM is immunogenic.

Key words: Streptococcus uberis, Streptococcus uberis adhesion molecule, sua gene sequencing, mastitis, dairy cow
\end{abstract}

\section{Short Communication}

Streptococcus uberis is an important environmental mastitis pathogen accounting for up to $33 \%$ of clinical mastitis in the United Kingdom (Hillerton et al., 1993). In the United States, S. uberis is isolated frequently from mammary glands of multiparous cows during the nonlactating period and causes a large proportion of streptococcal mastitis in heifers and multiparous cows during the peripartum period (Oliver, 1988; Leigh, 1999; Oliver et al., 2005). Streptococcus uberis mastitis has been and continues to be a major contributor to

Received October 22, 2013.

Accepted August 13, 2014.

${ }^{1}$ Corresponding author: ralmeida@utk.edu the estimated $\$ 2$ billion annual cost of mastitis in the United States.

To develop an effective vaccine for the control of S. uberis mastitis, knowledge of its virulence factors and their influence on pathogenesis is essential. Adherence, internalization, and intracellular persistence of $S$. uberis into bovine mammary epithelial cells (BMEC) are considered important virulence mechanisms for the establishment of IMI in dairy cows (Almeida and Oliver, 2006; Tamilselvam et al., 2006; Patel et al., 2009). Streptococcus uberis adhesion molecule (SUAM) is a key molecule in adherence to and internalization of $S$. uberis into BMEC in vitro (Almeida et al., 2006; Patel et al., 2009). Streptococcus uberis adhesion molecule has affinity for bovine lactoferrin (LF); a molecular bridge between SUAM-LF and a putative LF receptor on BMEC enhanced adherence and internalization of $S$. uberis into host cells (Patel et al., 2009).

The sua gene, encoding for the complete SUAM protein, was cloned, sequenced, and expressed as a recombinant protein in Escherichia coli (Prado et al., 2011). Bovine serum antibodies against recombinant SUAM (rSUAM) and pepSUAM, a 17-AA peptide located at the $\mathrm{N}$ terminus of SUAM, inhibited adherence to and internalization of $S$. uberis into mammary epithelial cells (Almeida et al., 2006; Prado et al., 2011).

The value of SUAM as a potential vaccine antigen to control S. uberis mastitis will depend on its conservation among strains of $S$. uberis. Objectives of the present study were to sequence and compare sua genes and their adjacent regions as well as detect the presence of SUAM by Western blot using serum from naturally Streptococcus uberis-infected cows in S. uberis strains isolated from diverse global locations.

Frozen stocks of strains of S. uberis isolated from cows with clinical or subclinical mastitis in the United States, United Kingdom, Australia, and New Zealand were grown in Todd Hewitt broth (THB; Difco Laboratories Inc., Detroit, MI) overnight at $37^{\circ} \mathrm{C}$ and genomic DNA was isolated using a Wizard Genomic DNA puri- 
Table 1. Primers for PCR and sequencing reactions ${ }^{1,2}$

\begin{tabular}{|c|c|c|}
\hline Sequence & Purpose & $\begin{array}{l}\text { Expected PCR } \\
\text { product size (bp) }\end{array}$ \\
\hline sua-U aaaatcttAAGACGTAGTGAACGCATGG & PCR and sequencing & 4,408 \\
\hline sua-D ccaagcttACTAACCAACGTAAACCTAA & PCR and sequencing & \\
\hline sua-D2 GTTCTTGCAAGTTGTGTCCG & Sequencing & \\
\hline sua-U3 CTAAAACTTGCTGAAGCAAC & Sequencing & \\
\hline sua-D3 ACTTTCATAATCTCTTGTGC & Sequencing & \\
\hline sua-3R ttaagcTTACACGTCCACCACGGATA & PCR & \\
\hline sua-1 gggaatTCATTTGGTAGGAGTGGCTGT & PCR & 2,971 \\
\hline sua-4 ggaagcTTGGTTGATATAGCACTTGGTG & PCR & \\
\hline sua-499 gotcgacggggggggoATTAGCACTCTTCAAG & Sequencing & \\
\hline sua-699 ggtcgacgggggggggGCTCCAAAAATCAGCA & Sequencing & \\
\hline
\end{tabular}

${ }^{1}$ Some primers were synthesized with a $5^{\prime}$ sequence tag (lowercase letters) to incorporate a particular restriction enzyme site.

${ }^{2}$ Sequences were obtained from Streptococcus uberis O140J strain genome sequence data published at http://www.sanger.ac.uk/Projects/ Suberis/ and S. uberis UT888 strain [National Center for Biotechnology Information (NCBI accession number D0232760; http://www.ncbi.nlm. nih.gov/genbank/).

fication kit (Promega Corp., Madison, WI). The primer pair sua-U and sua-D was used for PCR amplification of a 4.4-kb sua-containing DNA fragment, and primers used for sequencing are shown in Table 1.

The PCR reactions to amplify the 4.4-kb sua-containing DNA fragment were as follows: $50 \mu \mathrm{L}$ of Taq DNA polymerase reaction mix (Invitrogen Inc., Carlsbad, CA) with a final concentration of $1 \times$ High Fidelity PCR buffer, $200 \mu M$ concentrations of each deoxyribonucleotide triphosphate (dNTP), a $1.5 \mathrm{mM}$ concentration of $\mathrm{MgCl}, 0.3 \mu \mathrm{M}$ concentrations of each forward and reverse primer, approximately $100 \mathrm{ng}$ of genomic DNA, 1 unit of Platinum Taq DNA polymerase High Fidelity, and water. The PCR reaction conditions were as follows: cycle $1(1 \times): 95.0^{\circ} \mathrm{C}$ for $3 \mathrm{~min}$; cycle $2(30 \times)$ : $95.0^{\circ} \mathrm{C}$ for $30 \mathrm{~s}, 57.0^{\circ} \mathrm{C}$ for $30 \mathrm{~s}$, and then $68.0^{\circ} \mathrm{C}$ for 4.5 $\mathrm{min}$; and finally cycle $3(1 \times): 68.0^{\circ} \mathrm{C}$ for $10 \mathrm{~min}$. The PCR fragments were electrophoresed in a $0.8 \% \mathrm{E}$-Gel for DNA (Invitrogen Inc.) using a TrackIt $1 \mathrm{~kb}$ DNA ladder (Invitrogen Inc.) as molecular marker.

The sua-containing PCR fragments were sequenced using the Big Dye Terminator Sequencing kit v3.1 [Applied Biosystems Inc. (ABI), Foster City, CA]. Sequencing was run on an ABI 3730 sequencer. Sequence analysis and alignment were conducted using DNA Star Lasergene 8 software suite (DNASTAR Inc., Madison, WI). The ORF sequence of sua was codon optimized, synthesized, and cloned into the pET47b vector (kanamycin resistant) between restriction enzymes $N d e \mathrm{I}$ and EcoRI of the multiple cloning site (Blue Heron Biotechnology Inc., Bothell, WA). The sua insert (2,676 bp) was cut out and cloned into the pFLEX30 vector between NdeI and PstI sites. The plasmid (pFLEX/SUAM with kanamycin resistance marker) was enriched and puri- fied from E. coli $\mathrm{DH} 5 \alpha$, and transformed into E. coli BL21(DE3)/Star for expression of rSUAM. Recombinant SUAM was expressed as a fusion polypeptide (889 AA; $97,774 \mathrm{Da}$ ) containing $11 \mathrm{AA}$ of protective peptide fused into the $\mathrm{N}$ terminus of mature SUAM. Expression of rSUAM was induced by shifting the growth temperature to $42^{\circ} \mathrm{C}$ for $3 \mathrm{~h}$.

Bacterial proteins $(10 \mu \mathrm{g} /$ lane $)$ from 17 strains of S. uberis were electrophoresed on $10 \%$ polyacrylamide gels in the presence of SDS using Mini-PROTEAN 3 Cell (Bio-Rad Laboratories Inc., Hercules, CA) as previously described (Laemmli, 1970). Gels were either silver (Oakley et al., 1980) or Coomassie Brilliant Blue stained (CBB-G250; Fisher Scientific, Fair Lawn, NJ) as previously described (Neuhoff et al., 1988; Neumann, 1996). A third and fourth gel were transferred onto nitrocellulose membranes (Midwest Scientific Laboratory Equipment \& Supplies, Valley Park, MO) as previously described (Towbin et al., 1979) using a Trans-Blot SD Semi-Dry Electrophoresis Transfer Cell (Bio-Rad Laboratories Inc.). Membranes were blocked (5\% nonfat dry milk) and probed with rabbit anti-SUAM polyclonal serum $(1: 1,000)$ or bovine anti-S. uberis polyclonal serum $(1: 100)$ at room temperature for $1 \mathrm{~h}$. Blots were washed 3 times with PBS-0.05\% Tween-20 (pH 7.2), incubated with peroxidase-labeled goat anti-rabbit IgG (1:4,000; KPL Inc., Gaithersburg, MD) or horseradish peroxidase conjugated goat anti-bovine $\operatorname{IgG}(1: 500)$ horseradish peroxidase at room temperature for $1 \mathrm{~h}$, and color developed with tetramethylbenzidine (TMB) membrane substrate (KPL Inc.).

Using the primer pair sua-U and sua-D (Table 1), an approximately $4.4-\mathrm{kb}$ sua-containing fragment was PCR amplified from genomic DNA of all strains of $S$. 


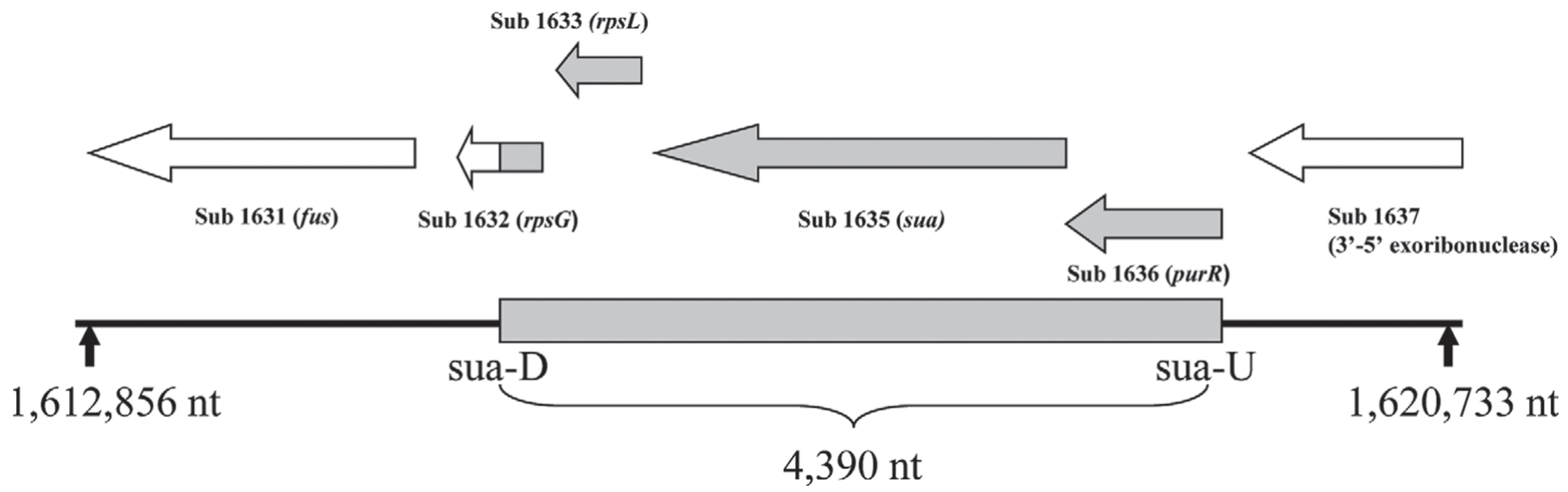

Figure 1. Sequenced region of sua-containing DNA fragment and its adjacent gene loci. The linear transcription map was drawn based on the complete genome sequence of Streptococcus uberis 0140J (GenBank accession no. AM946015; http://www.ncbi.nlm.nih.gov/genbank/). The 4.4-kb DNA fragments (marked in gray from sua-U to sua-D) amplified by PCR with the primer pair of sua-D and sua-U from genomic DNA samples of $S$. uberis strains was sequenced and compared. nt $=$ nucleotide.

uberis evaluated. The 4.4-kb PCR fragment contained sua [assigned as sub1635 in the completed S. uberis 0140J genome sequence; National Center for Biotechnology Information (NCBI) GenBank accession no. AM946015; http://www.ncbi.nlm.nih.gov/genbank/] with its flanking regions including purR (sub1636, DNA-binding transcriptional repressor) upstream, and rpsL (sub1633, 30S ribosomal subunit protein S12) and $\operatorname{rps} G$ (sub1632, 30S ribosomal subunit protein $\mathrm{S} 7$ ) downstream (Figure 1). Sequencing of the entire PCR fragment from S. uberis strains showed that sua $[2,718$ nucleotides (nt)] and its flanking regions (800 nt upstream and 500 nt downstream) were highly conserved. The percentage identity of deduced AA sequences (SUAM protein) of sua was $>97 \%$ (Table 2). Flanking regions of sua shared $>98 \%$ identities (data not shown).

The full length of the SUAM protein was probed with rabbit anti-SUAM serum (Figure 2) and all strains of S. uberis evaluated produced SUAM. The small peptide PepSUAM, located at the $\mathrm{N}$ terminus of the mature SUAM protein, is one of the surface exposed and immunogenic regions of SUAM (Almeida et al., 2010; Luther et al., 2010). The pepSUAM sequence was identical in 19 of 20 strains of $S$. uberis evaluated (DDMTTADQSPKLQGEEA) and only 1 AA difference (underscored) existed in the remaining strain of $S$. uberis (DDMTAAADQSPKLQGEEA).

Results from this investigation showed that a suacontaining fragment was PCR amplified from genomic DNA of all strains of $S$. uberis isolated from cows with S. uberis mastitis from the United States, Australia, New Zealand, and the United Kingdom. Luther et al. (2008) showed an approximately 2.6-kb sua-containing fragment in all strains of $S$. uberis evaluated. Differences in PCR fragment size reported in the present study and by Luther et al. (2008) were because of the PCR primers used. Using the primer pair sua-U and sua-D, the expected PCR fragment included sua and its flaking genes purR, rpsL, and part of rpsG (Figure $2)$, accounting for the larger PCR fragment size $(\sim 4.4$ $\mathrm{kb})$ reported in this investigation. The high identities of sua genes with flanking regions from strains of $S$. uberis isolated from geographically diverse areas of the world suggest that SUAM may be one of the adapted mechanisms used by $S$. uberis to infect and persist in the bovine mammary gland. Based on the high level of genetic variability of different strains of $S$. uberis, it has been difficult to identify a conserved antigenic molecule in S. uberis (Pullinger et al., 2006). Further evaluation of SUAM as a virulence factor will facilitate delineation of the importance of this molecule as a potential vaccine target.

During the last decade, studies on host-pathogen interactions showed that SUAM may be an important virulence factor involved in adherence to and internalization of $S$. uberis into BMEC, potentially leading to persistent $S$. uberis mastitis (Almeida et al., 2006; Tamilselvam et al., 2006). Under in vitro conditions, SUAM-mediated adherence of S. uberis to BMEC occurred through both LF-dependent and -independent mechanisms (Patel et al., 2009). Besides facilitating bacterial colonization, SUAM-dependent adherence may trigger internalization by a caveolae-dependent intracellular trafficking pathway in mammary epithelial cells, leading to persistence of $S$. uberis intracellularly and spreading of the pathogen to deeper tissues (Almeida and Oliver, 2006; Almeida et al., 2010). The same group demonstrated that bovine serum and milk containing anti-SUAM or anti-pepSUAM antibodies significantly inhibited adherence to and internalization 
Table 2. Percentage identity of deduced AA sequences of Streptococcus uberis adhesion molecule (SUAM) from 20 strains of $S$. uberis isolated from geographically diverse locations ${ }^{1}$

\begin{tabular}{|c|c|c|c|c|c|c|c|c|c|c|c|c|c|c|c|c|c|c|c|c|}
\hline \multirow[b]{2}{*}{ Strain } & \multicolumn{20}{|c|}{ Strain $^{2}$} \\
\hline & 1 & 2 & 3 & 4 & 5 & 6 & 7 & 8 & 9 & 10 & 11 & 12 & 13 & 14 & 15 & 16 & 17 & 18 & 19 & 20 \\
\hline 1 & - & 98.8 & 98 & 97.8 & 98.9 & 99.2 & 98 & 98.5 & 98 & 98 & 97.5 & 98.1 & 98.9 & 99 & 97.1 & 98.1 & 98.6 & 98.6 & 98.3 & 98.5 \\
\hline 2 & 1.1 & - & 98 & 97.8 & 98.7 & 98.8 & 98.2 & 98.2 & 98.2 & 98 & 97.6 & 98.1 & 99.8 & 98.8 & 97.5 & 98.1 & 98.3 & 99.7 & 98.1 & 98.2 \\
\hline 3 & 2.2 & 2.2 & - & 98.9 & 97.8 & 98.1 & 99.1 & 97.4 & 99.1 & 99 & 97 & 97 & 97.8 & 97.5 & 97.4 & 97 & 97.5 & 97.5 & 97.2 & 97.4 \\
\hline 4 & 2.1 & 2.1 & 1 & - & 97.9 & 98.2 & 99.4 & 97.5 & 99.4 & 100 & 97.1 & 97.1 & 97.9 & 97.6 & 97.2 & 97.1 & 97.6 & 97.6 & 97.4 & 97.5 \\
\hline 5 & 1 & 1.2 & 2.1 & 2 & - & 98.9 & 97.9 & 99.2 & 97.9 & 98 & 98.1 & 98.9 & 98.8 & 98.7 & 98 & 98.9 & 99.3 & 98.5 & 99.1 & 99.2 \\
\hline 6 & 0.7 & 1.1 & 1.8 & 1.7 & 1 & - & 98.5 & 98.5 & 98.5 & 98 & 97.8 & 98.1 & 98.9 & 99 & 97.6 & 98.1 & 98.6 & 98.8 & 98.3 & 98.5 \\
\hline 7 & 1.9 & 1.7 & 0.8 & 0.4 & 2 & 1.5 & - & 97.5 & 99.9 & 99 & 97.1 & 97.1 & 98.3 & 97.8 & 97.2 & 97.1 & 97.6 & 98 & 97.4 & 97.5 \\
\hline 8 & 1.5 & 1.7 & 2.6 & 2.5 & 0.7 & 1.5 & 2.5 & - & 97.5 & 97 & 98.3 & 99.4 & 98.3 & 98.7 & 97.5 & 99.2 & 99.8 & 98 & 99.8 & 99.9 \\
\hline 9 & 1.9 & 1.7 & 0.8 & 0.4 & 2 & 1.5 & 0 & 2.5 & - & 99 & 97.1 & 97.1 & 98.3 & 97.8 & 97.2 & 97.1 & 97.6 & 98 & 97.4 & 97.5 \\
\hline 10 & 2.2 & 2.2 & 0.7 & 0.3 & 2.1 & 1.8 & 0.8 & 2.6 & 0.8 & - & 97 & 97 & 97.8 & 97.5 & 97.4 & 97 & 97.5 & 97.5 & 97.2 & 97.4 \\
\hline 11 & 2.5 & 2.4 & 2.9 & 2.8 & 1.8 & 2.1 & 2.8 & 1.6 & 2.8 & 2.9 & - & 98.5 & 97.7 & 97.6 & 97.2 & 98.7 & 98.2 & 97.4 & 98.2 & 98.3 \\
\hline 12 & 1.8 & 1.8 & 2.9 & 2.8 & 1 & 1.8 & 2.8 & 0.4 & 2.8 & 2.9 & 1.5 & - & 98.2 & 98.6 & 97.4 & 99.4 & 99.3 & 97.9 & 99.3 & 99.4 \\
\hline 13 & 1 & 0.1 & 2.1 & 2 & 1.1 & 1 & 1.6 & 1.6 & 1.6 & 2.1 & 2.2 & 1.7 & - & 98.9 & 97.6 & 98.2 & 98.5 & 99.6 & 98.2 & 98.3 \\
\hline 14 & 0.9 & 1.1 & 2.5 & 2.4 & 1.2 & 0.9 & 2.1 & 1.2 & 2.1 & 2.5 & 2.4 & 1.3 & 1 & - & 97.1 & 98.3 & 98.8 & 98.6 & 98.6 & 98.7 \\
\hline 15 & 2.8 & 2.5 & 2.6 & 2.7 & 1.9 & 2.4 & 2.7 & 2.5 & 2.7 & 2.6 & 2.7 & 2.6 & 2.4 & 2.8 & - & 97.4 & 97.6 & 97.2 & 97.4 & 97.5 \\
\hline 16 & 1.8 & 1.8 & 2.9 & 2.8 & 1 & 1.8 & 2.8 & 0.7 & 2.8 & 2.9 & 1.2 & 0.4 & 1.7 & 1.6 & 2.6 & - & 99.1 & 97.9 & 99.1 & 99.2 \\
\hline 17 & 1.3 & 1.6 & 2.5 & 2.4 & 0.6 & 1.3 & 2.4 & 0.1 & 2.4 & 2.5 & 1.7 & 0.6 & 1.5 & 1.1 & 2.4 & 0.8 & - & 98.1 & 99.7 & 99.8 \\
\hline 18 & 1.3 & 0.2 & 2.5 & 2.4 & 1.5 & 1.1 & 1.9 & 1.9 & 1.9 & 2.5 & 2.6 & 2 & 0.3 & 1.3 & 2.7 & 2 & 1.8 & - & 97.9 & 98 \\
\hline 19 & 1.6 & 1.8 & 2.7 & 2.6 & 0.8 & 1.6 & 2.6 & 0.1 & 2.6 & 2.7 & 1.7 & 0.6 & 1.7 & 1.3 & 2.6 & 0.8 & 0.2 & 2 & - & 99.8 \\
\hline 20 & 1.5 & 1.7 & 2.6 & 2.5 & 0.7 & 1.5 & 2.5 & 0 & 2.5 & 2.6 & 1.6 & 0.4 & 1.6 & 1.2 & 2.5 & 0.7 & 0.1 & 1.9 & 0.1 & - \\
\hline
\end{tabular}

¿ $\quad{ }^{1}$ Percentage divergence in lower square; percentage similarity in upper square.

$\quad 21=$ O140J (United Kingdom); $2=$ UT888 (United States); $3=$ UT366 (United States); $4=$ UT102 (United States); $5=$ ATCC 13387 (United States); $6=$ UTM413 (United D States); $7=\mathrm{C} 2507$ (United States); $8=\mathrm{NZ1636H}$ (New Zealand); $9=$ W11020 (United States); $10=$ ATCC 27958 (United States); $11=$ PN09-2484-2 (New Zealand); $12=$ 으 PN09-2484-3 (New Zealand); $13=$ AU09-03953-4 (New Zealand); $14=$ AU-03953-5 (New Zealand); $15=$ PN09-02978-6 (New Zealand); $16=$ PN09-02978-7 (New Zealand); $17=$

VD4208 (Australia); $18=$ VD5239 (Australia); $19=$ VD4325 (Australia); $20=$ VD5586 (Australia) 


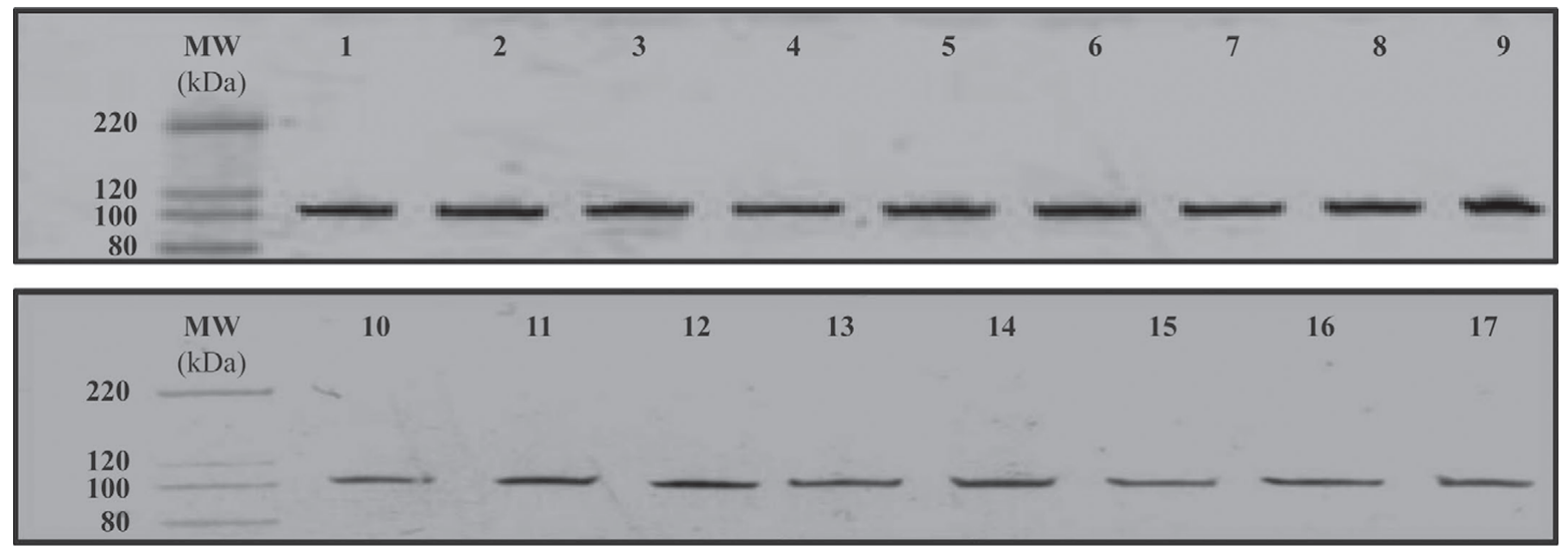

Figure 2. Western blot detecting Streptococcus uberis adhesion molecule (SUAM) from strains of S. uberis. Lane $1=$ UT888; lane 2 = PN09-02183; lane 3 = PN09-2484-2; lane 4 = PN09-2484-3; lane $5=$ AU09-03953-4; lane 6 = AU09-03953-5; lane 7 = PN09-02978-6; lane 8 = PN09-02978-7; lane 9 = CH09-05267; lane $10=0140 J$; lane $11=$ VD4208; lane 12 = VD5239; lane 13 = VD4325; lane 14 = VD5586; lane 15 =VD4433; lane $16=$ VD4203; lane $17=$ VD5181. Streptococcus uberis strain in lane 1 was isolated in the United States, strains in lanes 2 to 9 were isolated from New Zealand, the strain in lane 10 was isolated from the United Kingdom, and those in lanes 11 to 17 were isolated from dairy cows with mastitis in Australia.

of S. uberis into BMEC (Almeida et al., 2006). This observation was further confirmed by a study using a sua-gene deletion mutant strain of $S$. uberis UT888 (Chen et al., 2011).

Using 3 different prediction methods, the N-terminal pepSUAM (also designated as SUAM peptide I), 3 other regions in the middle portion of SUAM [peptide II (AA 342-356), peptide III (AA 357-371), and peptide IV (AA 439-453], and a region at the C-terminus of SUAM [peptide V (AA 705-719)] were predicted to be surfaced exposed B-cell epitopes (Almeida et al., 2011; Luther et al., 2010). Sequence data from this study confirmed $>93 \%$ identity in those epitopes among strains of $S$. uberis evaluated (data not shown). Further study of those regions may help to identify a SUAM-based strategy for the control of $S$. uberis mastitis in dairy cows.

In conclusion, results from this investigation demonstrated that a sua-containing fragment was PCR amplified from genomic DNA of all strains of $S$. uberis isolated from cows with $S$. uberis mastitis from the United States, Australia, New Zealand, and the United Kingdom. All of the strains evaluated produced SUAM, demonstrating that SUAM is ubiquitous and immunogenic in all strains of $S$. uberis evaluated thus far. The suggested putative role of SUAM in the pathogenesis of $S$. uberis mastitis together with the ubiquitous presence of SUAM in strains of $S$. uberis makes SUAM a promising candidate to be considered in the development of a vaccine for the control of $S$. uberis mastitis in dairy cows.

\section{ACKNOWLEDGMENTS}

This project was supported by a research agreement between The University of Tennessee (Knoxville) and Zoetis Veterinary Medicine Research and Development (VMRD; Kalamazoo, MI), by USDA (Washington, DC) Hatch funds, and by a grant from The University of Tennessee College of Veterinary Medicine Center of Excellence in Livestock Diseases and Human Health. We thank Douglas Luther (formerly of Department of Animal Science, The University of Tennessee, Knoxville)for his technical assistance.

\section{REFERENCES}

Almeida, R. A., J. R. Dunlap, and S. P. Oliver. 2010. Binding of host factors influences internalization and intracellular trafficking of Streptococcus uberis in bovine mammary epithelial cells. Vet. Med. Int. 2010:319192 http://dx.doi.org/10.4061/2010/319192.

Almeida, R. A., D. A. Luther, H. M. Park, and S. P. Oliver. 2006. Identification, isolation, and partial characterization of a novel Streptococcus uberis adhesion molecule (SUAM). Vet. Microbiol. 115:183-191.

Almeida, R. A., D. A. Luther, D. Patel, and S. P. Oliver. 2011. Predicted antigenic regions of Streptococcus uberis adhesion molecule (SUAM) are involved in adherence to and internalization into mammary epithelial cells. Vet. Microbiol. 148:323-328.

Almeida, R. A., and S. P. Oliver. 2006. Trafficking of Streptococcus uberis in bovine mammary epithelial cells. Microb. Pathog. 41:80-89.

Chen, X., O. Kerro Dego, R. A. Almeida, D. A. Luther, T. E. Fuller, and S. P. Oliver. 2011. Deletion of sua gene reduces the ability of Streptococcus uberis to adhere to and internalize into bovine mammary epithelial cells. Vet. Microbiol. 147:426-434.

Hillerton, J. E., M. F. H. Shearn, R. M. Teverson, S. Langridge, and J. M. Booth. 1993. Effect of pre-milking teat dipping on clinical mastitis on dairy farms in England. J. Dairy Res. 60:31-41. 
Laemmli, U. K. 1970. Cleavage of structural proteins during the assembly of the head of bacteriophage T4. Nature 227:680-685.

Leigh, J. A. 1999. Streptococcus uberis: A permanent barrier to the control of bovine mastitis? Vet. J. 157:225-238.

Luther, D. A., R. A. Almeida, and S. P. Oliver. 2008. Elucidation of the DNA sequence of Streptococcus uberis adhesion molecule gene (sua) and detection of sua in strains of Streptococcus uberis isolated from geographically diverse locations. Vet. Microbiol. 128:304-312.

Luther, D. A., R. A. Almeida, D. Patel, and S. P. Oliver. 2010. Surface region and epitope prediction of Streptococcus uberis adhesion molecule (SUAM). Page 706 in Mastitis Research into Practice, Proc. 5th IDF Mastitis Conf. J. E. Hillerton, ed. New Zealand Veterinary Association Foundation for Continuous Education, Christchurch, New Zealand.

Neuhoff, V., N. Arold, D. Taube, and W. Ehrhardt. 1988. Improved staining of proteins in polyacrylamide gel including isoelectric focusing gels with clear background at nanogram sensitivity using Coomassie Brilliant Blue G-250 and R-250. Electrophoresis 9:255-262.

Neumann, U. 1996. Quantitation of proteins separated by electrophoresis using Coomassie Brilliant Blue. Pages 173-178 in The Protein Protocol Handbook. J. M. Walker, ed. Humana Press, Totowa, NJ.

Oakley, B. R., D. R. Kirsch, and N. R. Morris. 1980. A simplified ultrasensitive silver stain for detecting proteins in polyacrylamide gels. Anal. Biochem. 105:361-363.

Oliver, S. P. 1988. Frequency of isolation of environmental mastitiscausing pathogens and incidence of new intramammary infections during the nonlactating period. Am. J. Vet. Res. 49:1789-1793.
Oliver, S. P., B. E. Gillespie, S. J. Headrick, M. J. Lewis, and H. H. Dowlen. 2005. Prevalence, risk factors and strategies for controlling mastitis in heifers during the periparturient period. Int. J. Appl. Res. Vet. Med. 3:150-162.

Patel, D., R. A. Almeida, J. R. Dunlap, and S. P. Oliver. 2009. Bovine lactoferrin serves as a molecular bridge for internalization of Streptococcus uberis into bovine mammary epithelial cells. Vet. Microbiol. 137:297-301.

Prado, M. E., R. A. Almeida, C. Ozen, D. A. Luther, M. J. Lewis, S. I. Headrick, and S. P. Oliver. 2011. Vaccination of dairy cows with recombinant Streptococcus uberis adhesion molecule induces antibodies that block adherence to and internalization of $S$. uberis into bovine mammary epithelial cells. Vet. Immunol. Immunopathol. 141:201-208.

Pullinger, G. D., M. López-Benavides, T. J. Coffey, J. H. Williamson, R. T. Cursons, E. Summers, J. Lacy-Hulbert, M. C. Maiden, and J. A. Leigh. 2006. Application of Streptococcus uberis multilocus sequence typing: Analysis of the population structure detected among environmental and bovine isolates from New Zealand and the United Kingdom. Appl. Environ. Microbiol. 72:1429-1436.

Tamilselvam, B., R. A. Almeida, J. R. Dunlop, and S. P. Oliver. 2006. Streptococcus uberis internalizes and persists in bovine mammary epithelial cells. Microb. Pathog. 40:279-285.

Towbin, H., T. Staehelin, and J. Gordon. 1979. Electrophoretic transfer of proteins from polyacrylamide gels to nitrocellulose sheets: Procedures and some applications. Proc. Natl. Acad. Sci. USA 76:4350-4354. 